\title{
Significance of interfaces in solid-state cells with porous electrodes of mixed ionic-electronic conductors
}

\author{
Meilin Liu*, Zhonglin Wu \\ School of Materials Science and Engineering, Georgia Institute of Technology, Atlanta, GA 30332-0245, USA
}

Received 9 October 1997; accepted 31 October 1997

\begin{abstract}
Solid-state cells based on different electrolyte materials and identical electrodes of a porous mixed ionic-electronic conductor (MIEC) are studied using impedance spectroscopy. It is found that impedance spectra of these cells are dramatically influenced by the properties of the electrolytes, implying that the electrolyte-electrode interfaces, including the triple-phase boundaries (TPBs), are important reaction sites in these cells and can not be ignored, in general, in modelling the electrode kinetics of porous MIEC electrodes. Further, the observed effect of electrical state of an MIEC electrode (or an applied voltage across the cell) on cell impedance conclusively confirms that the nature of the rate-limiting step(s) of the electrode reactions occurring in such a cell is electrochemical; it is not true, in general, that the electrode kinetics of such a cell are determined merely by pure chemical processes such as 'surface exchange' and oxygen diffusion.
\end{abstract}

Keywords: Impedance spectroscopy; Triple-phase boundary; Mixed conductors; Electrode-electrolyte interface; MIEC electrode

\section{Introduction}

While mixed ionic-electronic conductors (MIECs) have been widely studied as electrodes for solid-state cells, misconceptions on reaction paths and reaction mechanisms in such a cell still exist [1,2]. Equivalent circuit analysis [1] indicates that the MIEC/gas interface is not the only active site for electrode reaction to occur in such a cell; oxygen reduction and evolution may also occur at the triple-phase boundary (TPB) among the electrolyte, the MIEC, and $\mathrm{O}_{2}$ gas, and the TPB among the current collector, the MIEC, and $\mathrm{O}_{2}$ gas. These TPBs are other possible reaction paths parallel to the $\mathrm{MIEC} /$ gas

\footnotetext{
*Corresponding author. Tel.: + 1 (404) 894-6114; e-mail: meilin.liu@mse.gatech.edu
}

interface in solid-state cells with porous MIEC electrodes. The relative significance of each path is determined not only by the transport and catalytic properties of the MIEC but also by the interfaces between the MIEC and other cell components (including the TPBs). Under the conditions that (i) the thickness of the MIEC electrode, $L$, is much greater than the electrochemically-active layer of the electrode, $\delta$, and (ii) the MIEC has overwhelming electronic conductivity, the total impedance of such a cell can be expressed as [1]

$Z_{\text {cell }}=R_{\mathrm{i}, \mathrm{el}}+2\left[Z_{\mathrm{ee}}+\frac{Z_{\mathrm{TPBI}}\left(Z_{\mathrm{rt}}+Z_{\mathrm{ii}}\right)}{Z_{\mathrm{TPBI}}+\left(Z_{\mathrm{rt}}+Z_{\mathrm{ii}}\right)}\right]$ 


$$
\frac{Z_{\text {cell }}-R_{\mathrm{i}, \mathrm{el}}}{2}=Z_{\mathrm{ee}}+Z_{\mathrm{TPBI}}
$$

when the diffusion of oxygen in the MIEC and the reaction at the MIEC/gas interface are much slower than any other processes occurring in the cell. The effectiveness of the MIEC/gas interface, characterized by $1 / Z_{\mathrm{rt}}$, is influenced by the rates of several sequential processes: transfer of ionic defects across the MIEC/electrolyte interface, transport of ionic and electronic defects in the MIEC, reaction at the $\mathrm{MIEC} /$ gas interface, and transport of $\mathrm{O}_{2}$ gas in the pores of the MIEC. In general, therefore, oxygen diffusion in the MIEC and the reaction at the MIEC/ gas interface are only part, not the entirety, of the electrode process in such as cell; in particular, the contributions of solid-state diffusion and surface reaction to cell impedance become negligible [2], rather than dominating, when the impedances arising from these two processes are sufficiently greater than the impedance of TPBs. The significance of TPBs in other solid-state cells is also emphasized in several recent publications [3-5].

Eq. (1) clearly indicates that $Z_{\text {cell }}$ may be influenced not only by the properties of the MIEC but also by the properties of the electrolyte and the interfaces (including the TPBs). Under certain conditions, in fact, $Z_{\text {cell }}$ is determined only by the properties of the interfaces. Further, Eq. (1) predicts that $Z_{\text {cell }}$ may be influenced by the electrical state of the MIEC electrode; in particular, the $Z_{\text {TPBI }}$ in Eq. (1) may be reduced by an applied DC bias across the cell due to field-assisted charge transfer as anticipated from the electrochemical nature of the interfaces (or Butler-Volmer equation). The latter effect can be used to determine if the nature of the ratelimiting step(s) of the electrode processes is chemical or electrochemical.

In order to examine the effect of interfaces and TPBs on electrode kinetics, impedance responses of solid-state cells based on different electrolyte materials and an identical MIEC electrode material are investigated. Further, the effect of the electrical state of the MIEC on cell impedance are characterized to determine the nature of the rate-limiting step(s) of the electrode reactions. These results are important to correct understanding and meaningful modelling of porous MIEC electrodes in solid-state cells.

\section{Experimental}

Electrolyte materials studied were yttria-stabilized bismuth oxide $\mathrm{Bi}_{1.5} \mathrm{Y}_{0.5} \mathrm{O}_{3}$ (YSB), gadolinia-doped barium cerate $\mathrm{BaCe}_{0.8} \mathrm{Gd}_{0.2} \mathrm{O}_{3}$ (BCG), strontia-stabilized ceria $\mathrm{Ce}_{0.9} \mathrm{Sr}_{0.1} \mathrm{O}_{2}$ (SSC), and yttria-stabilized zirconia $\mathrm{Zr}_{0.92} \mathrm{Y}_{0.08} \mathrm{O}_{2}$ (YSZ). The MIEC electrode materiel studied was $\mathrm{La}_{0.7} \mathrm{Sr}_{0.3} \mathrm{Co}_{0.7} \mathrm{Fe}_{0.3} \mathrm{O}_{3}$ (LSCF). Powders of YSB [6]. BCG [7], SSC [8], and LSCF [9] were prepared as described in the literature and YSZ powder was obtained from Tosho Corporation. Electrolyte pellets of YSB, BCG, SSC, and YSZ were formed by uniaxial and isostatic pressing, followed by sintering at $900^{\circ} \mathrm{C} / 4 \mathrm{~h}, 1550^{\circ} \mathrm{C} / 8 \mathrm{~h}$, $1550^{\circ} \mathrm{C} / 8 \mathrm{~h}$, and $1100^{\circ} \mathrm{C} / 4 \mathrm{~h}$, respectively, to obtain sintered relative density greater than $95 \%$. LSCF powder was mixed with $\mathrm{V}-006$ binder (Heraeus) in a weight ratio of 7 to 3 , together with $35 \mathrm{wt} \%$ turpentine and $5.7 \mathrm{wt} \%$ peanut oil, through ball milling to obtain paste of LSCF electrode, which was screen printed on electrolyte pellets and then fired at $900^{\circ} \mathrm{C}$ for $0.5 \mathrm{~h}$. The thicknesses of the porous LSCF electrodes after firing varied from 10 to $20 \mu \mathrm{m}$. The microstructures of the prepared electrolyte and porous LSCF electrodes were examined using an SEM. Silver mesh was used as current collector and the cell impedances were measured as described elsewhere [10].

\section{Results and discussion}

\subsection{Microstructure of the porous electrodes}

Shown in Fig. 1 is a cross-sectional view of a cell consisting of a YSB electrolyte and two porous LSCF electrodes, i.e., a LSCF/YSB/LSCF cell. The estimated porosity of the electrode is about $22 \%$. The microstructures of the LSCF electrodes prepared on other electrolytes are similar; there are no statistically significant differences in microstructures observed among the LSCF electrodes processed on different electrolytes.

\subsection{Effect of electrical states}

Shown in Fig. 2 are some effects of the amplitude of an applied DC bias on the impedance response of 


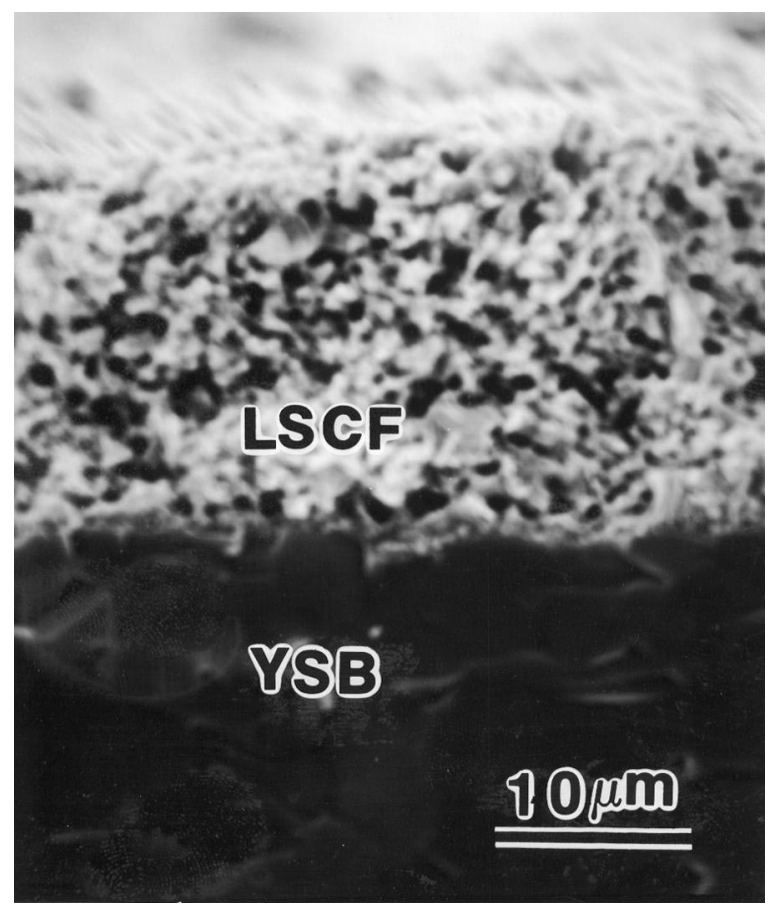

Fig. 1. An SEM micrograph showing a cross-sectional view of an LSCF electrode and an LSCF/YSB interface, from a cell consisting of a YSB electrolyte and two porous LSCF electrodes.

a cell based on a BCG electrolyte measured in air at $700^{\circ} \mathrm{C}$. In this experiment, the only variables were the electrical states of the two MIEC electrodes in the cell while all other conditions were kept exactly the same. It can be clearly seen that the polarization impedance of the electrodes (i.e., the impedance arising from electrode reactions at $\mathrm{MIEC} /$ gas interface and at the two types of TPBs), $Z_{\text {cell }}-R_{\mathrm{b}}$,

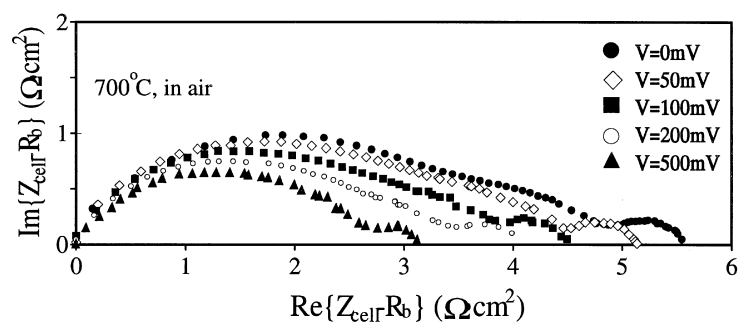

Fig. 2. Effect of the amplitude of an applied DC bias on the impedance response of a cell consisting of a BCG electrolyte and two porous LSCF electrodes measured in air at $700^{\circ} \mathrm{C}$. The thicknesses of the porous LSCF electrodes were about $20 \mu \mathrm{m}$. The voltages represent the amplitude of the applied DC bias. decreased with the amplitude of the applied DC bias, indicating that the rate-limiting step of the electrode reaction occurring in the cell is an electrochemical, not a pure chemical, reaction. Evidently, only the impedance to a charge-transfer, or an electrochemical reaction, may be reduced by an applied voltage as predicted by Butler-Volmer equation. Thus, it is not true in general that the electrode kinetics of cells with porous MIEC electrodes are determined merely by chemical processes (such as 'surface exchange' and oxygen diffusion) [2].

These experimental results are consistent with Eq. (1), in which $Z_{\mathrm{ee}}, Z_{\mathrm{ii}}, Z_{\mathrm{rt}}$, and $Z_{\mathrm{TPB}}$ are all electrochemical in nature and should depend in general on the electrical state of the electrode. Although the effect of electrical potential on $Z_{\mathrm{rt}}$ can be small under certain conditions, $Z_{\mathrm{rt}}$ should be considered electrochemical in general ${ }^{1}$. This is because an applied voltage across an MIEC may influence the distribution of the internal electric field and the distribution of defects in the MIEC and near the MIEC/ gas interface [11]; consequently, it may influence the resistance arising from charge transfer across the $\mathrm{MIEC} /$ gas interface or the reaction kinetics.

\subsection{Effect of electrolyte materials}

Shown in Fig. 3 are some typical impedance spectra of cells based on identical LSCF electrodes and different electrolyte materials measured in air at different temperatures. It is important to note that all cells had the same electrode material and all electrodes were processed in an identical manner to minimize the variations in microstructures of the electrodes due to processing. Accordingly, while the nature of the electrolyte-electrode interfaces (including the TPBs) of each cell was altered by changing the electrolyte material, the bulk properties of the MIEC electrodes (including the reaction at the MIEC/gas interface and the transport of defects in the MIEC) of all cells should remain unchanged since the material and the processing of all electrodes were the same.

\footnotetext{
${ }^{1}$ The reaction at the MIEC/gas interface may be considered a chemical reaction when the effect of an electrical potential is insignificant (e.g., when the electronic conductivity of the MIEC is overwhelming compared to its ionic conductivity).
} 

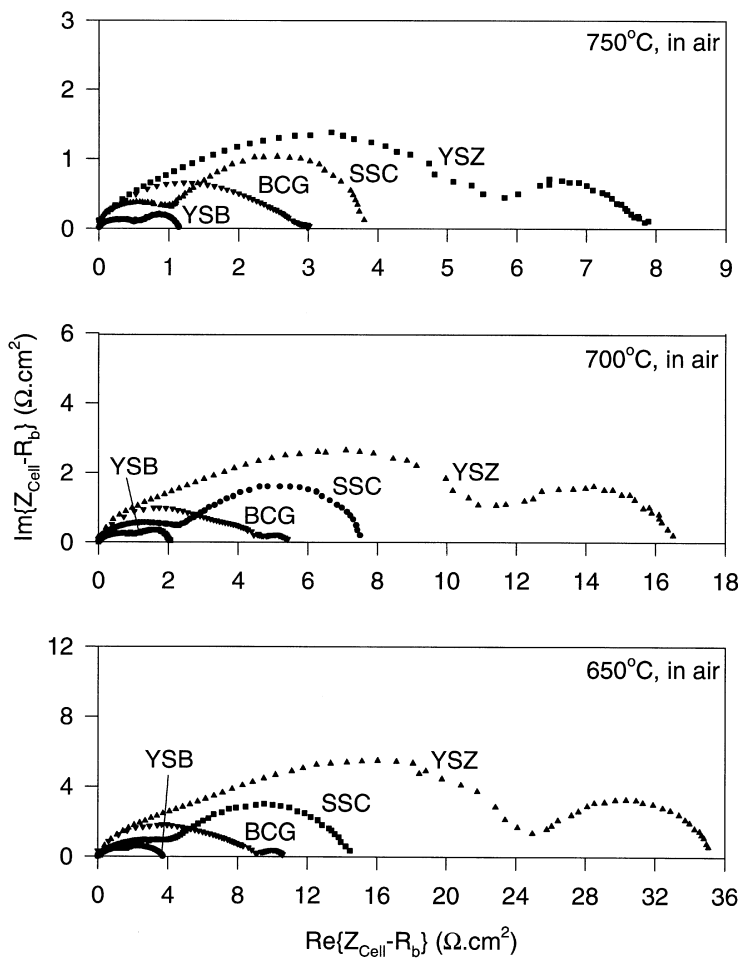

Fig. 3. Typical impedance spectra of cells based on different electrolytes and identical LSCF electrodes measured in air at three different temperatures: (a) $750^{\circ} \mathrm{C}$, (b) $700^{\circ} \mathrm{C}$, and (c) $650^{\circ} \mathrm{C}$. All electrodes were processed in an identical manner with electrode thickness of about $20 \mu \mathrm{m}$. The thickness of YSB, BCG, SSC, and YSZ electrolyte was $1.37 \mathrm{~mm}, 0.95 \mathrm{~mm}, 1.63 \mathrm{~mm}$, and $0.87 \mathrm{~mm}$, respectively.

The bulk and the total resistance of each cell, $R_{\mathrm{b}}$ and $R_{\mathrm{T}}$ [12], can be readily determined from the intercepts of the impedance loops with the real axis at high and low frequencies, respectively. Thus, the polarization resistances of the electrodes in each cell can be estimated as $R_{\mathrm{P}}=R_{\mathrm{T}}-R_{\mathrm{b}}$, when the electronic transference number of the electrolyte is negligible; otherwise the $R_{\mathrm{P}}$ may be calculated using equations discussed elsewhere [12]. It can be seen from Fig. 3 that the resistances relevant to the polarization resistance of the electrodes, $R_{\mathrm{T}}-R_{\mathrm{b}}$, are influenced dramatically by the electrolyte materials used in each cell. At $750^{\circ} \mathrm{C}$, for instance, $R_{\mathrm{T}}-R_{\mathrm{b}}$ varied approximately from about $1.15 \Omega \mathrm{cm}^{2}$ for a cell with a YSB electrolyte, to about $3.0 \Omega \mathrm{cm}^{2}$ for a cell with a BCG electrolyte, to about $3.7 \Omega \mathrm{cm}^{2}$ for a cell with a SSC electrolyte, and to about $8.0 \Omega \mathrm{cm}^{2}$ for a cell with a YSZ electrolyte. Further, the shapes of the impedance spectra are quite different for cells with different electrolyte materials. All these observations consistently suggest that the impedances of these cells are significantly influenced by the interfaces between the electrolyte and the electrodes (including TPBs), not determined just by the bulk properties of the MIEC (surface exchange and oxygen diffusion) [2]. If the electrode kinetics are indeed dominated only by the reaction at MIEC/gas interface and the transport of defects in the MIEC, the polarization impedance of the electrodes, $Z_{\text {cell }}-R_{\mathrm{b}}$, of all cells studied at each temperature should be independent of electrolyte material; the impedance spectra acquired at each temperature should be approximately the same, not vary up to about 7 times as shown in Fig. 3.

One concern is that the microstructure, and hence the impedance response, of the LSCF electrodes may vary from cell to cell due to variations in processing of the electrodes. To determine the 'spread' in microstructure (and, hence, variation in impedance response) of the porous LSCF electrodes due to processing, four cells based on dense BCG electrolytes and porous LSCF electrodes were processed in a similar manner. Shown in Fig. 4 are the impedance spectra of the four cells measured in air at $700^{\circ} \mathrm{C}$ under identical conditions. Of the 4 cells studied, the relative error of the resistance, $R_{\mathrm{T}}-R_{\mathrm{b}}$, is about $23 \%$, indicating that the 'spread' in impedance due to processing of the porous LSCF electrodes $(23 \%)$ is much smaller than the observed variation in impedance due to change of electrolyte (up to 7 times).

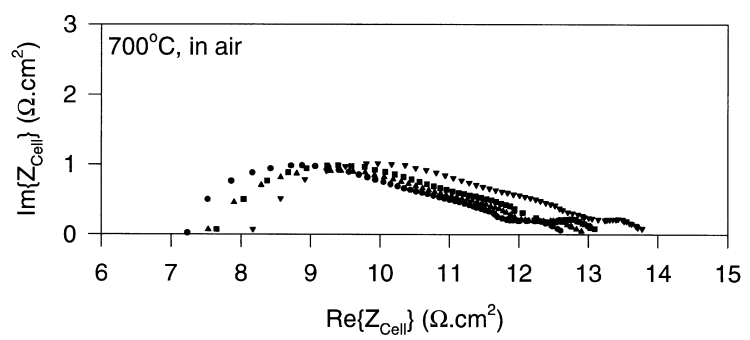

Fig. 4. Impedance spectra of four cells based on BCG electrolyte and LSCF electrode, each being prepared using similar processing procedures. The BCG electrolyte thickness varied from $0.92 \mathrm{~mm}$ to $1.06 \mathrm{~mm}$ and the LSCF electrode thickness varied from 10 to $20 \mu \mathrm{m}$. 
One may argue, however, that the change of electrolyte may also alter the chemical composition and microstructure of the MIEC in intimate contact with the electrolyte. Electron microscopy of crosssectional views of the cells suggests that there is no statistically significant differences in microstructures of LSCF electrodes prepared on different electrolytes. Although it is difficult to quantify these effects experimentally, the observed variations in interfacial impedance (e.g., increased about 7 times when the electrolyte was changed from YSB to YSZ) are too great to be attributed just to these effects. These observations, once again, strongly suggest that the interfaces between electrolyte and electrodes (including the TPBs) play an important role in the electrode kinetics of these cells, as implied by Eq. (1). In fact, Eq. (1) would predict that cells with identical MIEC electrodes but with different electrolyte materials should have different electrode impedance $\left(Z_{\text {cell }}-R_{\mathrm{b}}\right)$ simply because the nature of the interfaces and TPBs depends critically on the electrolyte in contact with the MIEC electrodes.

\section{Conclusion}

The observed dramatic dependence of cell impedance on electrolyte materials strongly suggests that the interfaces between the electrolyte and the electrode, particularly the TPBs, are an important part of the reaction zone in such a cell. It also implies that the use of MIECs as electrodes in solid-state cells may not only extend the reaction zone to the MIEC/ gas interface but also alter the catalytic activity of the triple-phase boundary (TPB) between the electrode, $\mathrm{O}_{2}$ gas, and the electrolyte (or current collector). Further, the observed dependence of cell impedance on the electrical states of the MIEC electrodes conclusively confirms that the rate-limiting step of the electrode reaction is an electrochemical rather than a pure chemical reaction; it is not true in general that pure chemical processes are the only processes which dominate the kinetics of such a cell. Accordingly, electrolyte-electrode interfaces (particularly the TPBs) must be carefully considered in order to model porous MIEC electrodes correctly. Proper incorporation of interfaces and TPBs into boundary conditions in modelling cells with porous MIEC electrodes will be discussed in a subsequent publication [13].

\section{List of symbols}

$L \quad$ thickness of a porous MIEC electrode, $\mathrm{cm}$

$R_{\mathrm{b}} \quad$ bulk resistance of a cell $\left(\lim _{\omega \rightarrow 0} Z_{\text {cell }} \approx R_{\mathrm{b}}\right)$, $\Omega \mathrm{cm}^{2}$

$R_{\mathrm{i}, \text { el }} \quad$ resistance to the motion of ionic defects in the electrolyte, $\Omega \mathrm{cm}^{2}$

$R_{\mathrm{P}} \quad$ interfacial polarization resistance of a cell, $\Omega \mathrm{cm}^{2}$

$R_{\mathrm{T}} \quad$ total DC resistance of a cell $\left(\lim _{\omega \rightarrow 0} Z_{\text {cell }} \approx\right.$ $\left.R_{\mathrm{b}}\right), \Omega \mathrm{cm}^{2}$

$Z_{\text {cell }} \quad$ total cell impedance, $\Omega \mathrm{cm}^{2}$

$Z_{\text {ee }}$ impedance to electron transfer across cc/ MIEC interface, $\Omega \mathrm{cm}^{2}$

$Z_{\mathrm{ii}} \quad$ impedance to ion transfer across el/MIEC interface, $\Omega \mathrm{cm}^{2}$

$Z_{\mathrm{rt}} \quad$ impedance to reaction at MIEC/gas interface and to the associated transport of the species involved in the reaction, $\Omega \mathrm{cm}^{2}$

$Z_{\text {TPBI }}$ impedance to the reaction at TPB among the electrolyte, the MIEC, and $\mathrm{O}_{2}$ gas (including the impedance arising from the associated mass transfer), $\Omega \mathrm{cm}^{2}$

$\delta$ thickness of the electrochemically-active layer of a porous MIEC electrode, $\mathrm{cm}$

\subsection{Abbreviations}

BCG gadolinia-doped barium cerate,

LSCF $\quad \mathrm{La}_{0.7} \mathrm{Sr}_{0.3} \mathrm{Co}_{0.7} \mathrm{Fe}_{0.3} \mathrm{O}_{3}$

MIEC mixed ionic-electronic conductor

SSC strontia-stabilized ceria, $\mathrm{Ce}_{0.9} \mathrm{Sr}_{0.1} \mathrm{O}_{2}$

TPB triple-phase boundary

YSB yttria-stabilized bismuth oxide, $\mathrm{Bi}_{1.5} \mathrm{Y}_{0.5} \mathrm{O}_{3}$

YSZ yttria-stabilized zirconia, $\mathrm{Zr}_{0.92} \mathrm{Y}_{0.08} \mathrm{O}_{2}$

\section{Acknowledgements}

The authors would like to acknowledge Professor Jack Winnick for valuable discussions. This work was supported by NSF (Award No. DMR-9357520) 
and EPRI (Contract No. RP 1676-19) and their financial support of this research is gratefully acknowledged.

\section{References}

[1] M. Liu, J. Electrochem. Soc. 145 (1998) 142.

[2] M. Liu, J. Winnick, J. Electrochem. Soc. 144 (1997) 1881.

[3] M. Kleitz, F. Petitbon, Solid State Ionics 92 (1996) 65.

[4] F.H. van Heuveln, H.J.M. Bouwmeester, F.P.F. van Berkel, J. Eletrochem. Soc. 144 (1997) 134.

[5] J. Jamnik, J. Maier, Solid State Ionics 94 (1997) 189.

[6] M.J. Verkerk, K. Keizer, A.J. Burggraaf, J. Appl. Electrochem. 10 (1980) 81.
[7] W. Rauch, M. Liu, in: P.N. Kumta, G.S. Rohrer, U. Balachandran (Eds.), Role of Ceramics in Advanced Electrochemical Systems, The American Ceramic Society, Westerville, OH, Ceramic Transitions, 65, 1995, pp. 73-83.

[8] R.G. Anderson, A.S. Nowick, Solid State Ionics 53-56 (1981) 547.

[9] Y. Ohno, S. Nagata, H. Sato, Solid State Ionics 3-4 (1981) 439.

[10] M. Liu, W. Rauch, H. Hu, in: H. Anderson, A. Khandkar, M. Liu (Eds.), Proceedings of the First International Symposium on Ceramic Membranes, The Electrochemical Society, Pennington, NJ, vol. 95-24, 1996, pp. 192-220.

[11] M. Liu, J. Electrochem. Soc. 144 (1997) 1813.

[12] M. Liu, H. Hu, J. Electrochem. Soc. 143 (1996) L109.

[13] Z.L. Wu, M. Liu, Solid State Ionics, to be submitted. 